\title{
MICU1 protects against myocardial ischemia/reperfusion injury and its control by the importer receptor Tom70
}

\author{
Qiang Xue ${ }^{1}$, Haifeng Pei ${ }^{\star * 2}$, Qinshe Liư ${ }^{3}$, Mingjun $\mathrm{Zhao}^{3}$, Jing Sun ${ }^{3}$, Erhe Gao ${ }^{4}$, Xinliang $\mathrm{Ma}^{5}$ and Ling Tao,
}

Mitochondrial $\mathrm{Ca}^{2+}$ overload is a main contributor to mitochondrial damage hence cardiomyocyte death in myocardial ischemia/ reperfusion (MI/R) injury. MICU1 has been recently identified as an important regulator of mitochondrial $\mathrm{Ca}^{2+}$ homeostasis. Here we try to identify the role of MICU1 in MI/R, and to investigate whether the mitochondrial importer receptor Tom70 possesses critical roles in the mitochondrial translocation of MICU1 and MI/R. Specific small interfering RNA $(20 \mu \mathrm{g})$ against MICU1 and Tom70, and lentivirus vectors carrying the Tom70a sequences $\left(3.3 \times 10^{7} \mathrm{TU}\right)$ were delivered through intramyocardial injection. Seventy-two hours after injection, mice were subjected to $30 \mathrm{~min}$ of MI followed by $3 \mathrm{~h}$ (for cell apoptosis and mitochondrial damage assessment) or $24 \mathrm{~h}$ (for cardiac function and infarct size determination) of reperfusion. MI/R had no significant effect on total MICU1 expression, but caused significant reduction of MICU1 in mitochondria. Knockdown of MICU1 significantly aggravated MI/R injury, as evidenced by enlarged infarct size, depressed cardiac function and increased myocardial apoptosis. Moreover, MICU1 deficiency resulted in markedly aggravated mitochondrial $\mathrm{Ca}^{2+}$ overload, consequently destructed mitochondrial morphology and suppressed mitochondrial function (evidenced by decreased ATP production). Interestingly, mitochondrial Tom70 was also decreased in MI/R. Genetic loss-function study revealed that mitochondrial MICU1 expression was depressed by Tom70 ablation. Furthermore, Tom70 deficiency significantly aggravated MI/R injury and worsened mitochondrial $\mathrm{Ca}^{2+}$ overload. However, supplementation of Tom70 significantly attenuated MI/R injury, preserved mitochondrial morphology and function, and inhibited mitochondrial $\mathrm{Ca}^{2+}$ overload, all of which were abolished by MICU1 suppression. Mitochondrial Tom70/MICU1 pathway protects against MI/R injury, in which mitochondrial localization of MICU1 is governed by Tom70, and MICU1 serves as an indispensable factor in Tom70's cardioprotection.

Cell Death and Disease (2017) 8, e2923; doi:10.1038/cddis.2017.280; published online 13 July 2017

Reperfusion strategies with the use of thrombolytic agents and primary percutaneous coronary intervention is undoubtedly beneficial in myocardial infarction (MI), however, they also cause irreversible detrimental effects termed myocardial reperfusion injury. ${ }^{1,2}$ Identifying novel therapeutic interventions reducing reperfusion injury may increase survival rate and ultimately reduce death rate caused by MI.

The structure and biochemical functions of mitochondria, the primary source of ATP supply in the contracting cardiac myocytes and the 'headquarter' of apoptotic cell death, are the major targets of ischemia/reperfusion (I/R) injury. ${ }^{3-5}$ Mitochondrial $\mathrm{Ca}^{2+}$ homeostasis has an important role in the maintenance of a variety of cellular functions. ${ }^{6} \mathrm{Ca}^{2+}$ is central to the cardiac excitation-contraction coupling and the signaling networks that regulate pathological myocardial growth and remodeling. ${ }^{7}$ Accumulating evidence show that mitochondrial $\mathrm{Ca}^{2+}$ overload is associated with mitochondrial dysfunction, contractile dysfunction and cell death. ${ }^{8,9}$ Complete understanding of the molecular mechanisms leading to the elevation of mitochondrial $\mathrm{Ca}^{2+}$ content in post-MI cardiomyocytes thus may hold great promise in attenuating myocardial ischemia/reperfusion ( $\mathrm{Ml} / \mathrm{R})$ injury.
Recent experimental evidence indicates that $\mathrm{Ca}^{2+}$ handling at mitochondrial level is more tightly controlled by the balance between molecules that stimulate mitochondrial $\mathrm{Ca}^{2+}$ uptake and molecules that inhibit $\mathrm{Ca}^{2+}$ uptake. ${ }^{10}$ Specifically, the mitochondrial $\mathrm{Ca}^{2+}$ uniporter (MCU) is the major molecule stimulating mitochondrial $\mathrm{Ca}^{2+}$ uptake. ${ }^{11}$ With an in vivo $\mathrm{MI} / \mathrm{R}$ model, Luongo et al. ${ }^{12}$ revealed that $\mathrm{MCU}$ deficiency conferred resistance to IR injury by preventing mitochondrial $\mathrm{Ca}^{2+}$ overload. Moreover, mitochondrial $\mathrm{Ca}^{2+}$ uptake 1 (MICU1) was recently identified as a molecule localized to the inner mitochondrial membrane to regulate mitochondrial $\mathrm{Ca}^{2+}$ uptake. ${ }^{13}$ The MICU1 serves as an inhibitory effecter for mitochondrial $\mathrm{Ca}^{2+}$ influx with localization to the inner mitochondrial membrane. ${ }^{14}$ These two molecules biochemically interact with each other, and they are co-expressed across tissues and species. ${ }^{15}$ Mallilankaraman et al. ${ }^{16}$ found that MICU1 was an essential inhibitory gatekeeper for MCU-mediated mitochondrial $\mathrm{Ca}^{2+}$ uptake that regulated cell survival. MICU1 is required to preserve normal mitochondrial $\mathrm{Ca}^{2+}$ level under basal conditions. In its absence, mitochondria become constitutively loaded with $\mathrm{Ca}^{2+}$ through

\footnotetext{
${ }^{1}$ Department of Cardiology, Xijing Hospital, Fourth Military Medical University, Xi'an 710032, China; ${ }^{2}$ Department of Cardiology, Chengdu Military General Hospital, Chengdu 610083, China; ${ }^{3}$ Shaanxi University of Chinese Medicine, Xi'an 712046, China; ${ }^{4}$ Center of Translational Medicine, Temple University School of Medicine, Philadelphia, PA 19140, USA and ${ }^{5}$ Department of Emergency Medicine, Thomas Jefferson University, Philadelphia, PA 19107, USA

${ }^{*}$ Corresponding author: L Tao, Department of Cardiology, Xijing Hospital, Fourth Military Medical University, 15 Changlexi Road, X'an 710032 , China. Tel: +86 29 8477 5183; Fax: +86 298321 1024; E-mail: xijingheart@ 163.com

or H Pei, Department of Cardiology, Chengdu Military General Hospital, 270 Tianhui Road, Chengdu 610083, China. Tel: +86 2886570211 ; Fax: +86 2883570341 ; E-mail: web2010@foxmail.com
}

Received 04.3.17; revised 21.4.17; accepted 15.5.17; Edited by S Lavandero 
a

Mouse cardiac tissues

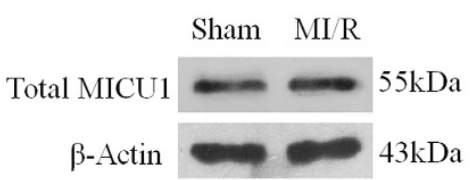

b

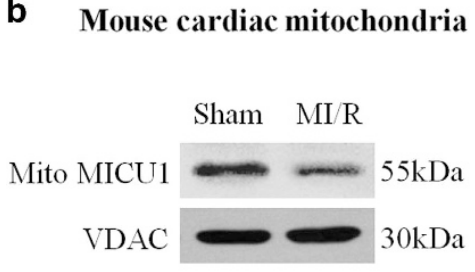

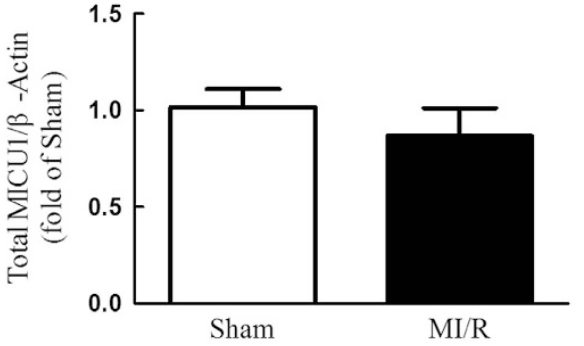

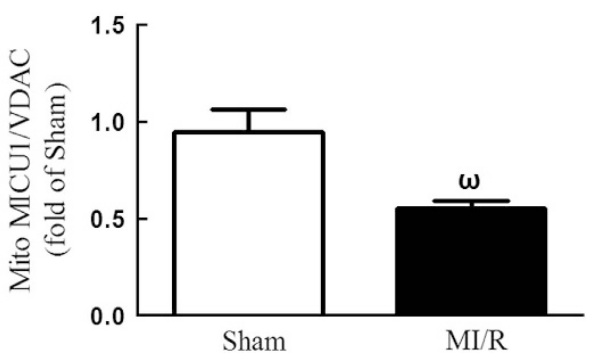

Figure 1 MICU1 responded to MI/R injury. (a) Representative western blot images showing total MICU1 expression using $\beta$-actin as loading control in the left panel; intensities of total MICU1 relative to $\beta$-actin in the right panel. (b) Representative western blot images showing mitochondrial MICU1 expression using VDAC as loading control in the left panel; intensities of mitochondrial MICU1 relative to VDAC in the right panel. Mito, mitochondria; VADC, voltage-dependent anion channel. Presented values are means \pm S.E.M. $N=6-8 /$ group. ${ }^{\omega} P<0.05$ versus sham

MCU, triggering excessive reactive oxygen species generation and increasing sensitivity to apoptotic stress. ${ }^{16}$ However, whether $\mathrm{MI} / \mathrm{R}$ may alter MICU1 expression/function, thus contributing to post-Ml mitochondrial $\mathrm{Ca}^{2+}$ overload and subsequent cell death, have not been previously investigated.

Although mitochondria possess their own genome that encodes for 13 essential subunits of the oxidative phosphorylation system, the majority of $\sim 1500$ mitochondrial proteins are synthesized in the cytosol and then transported into corresponding compartments in mitochondria. Breakthroughs on mitochondrial protein targets demonstrate that translocases of outer membrane (Tom) complex is responsible for initial recognition of mitochondrial pre-proteins from the cytosol. ${ }^{17}$ As an important receptor of the Tom machinery, Tom70 preferentially binds the internal targeting signals of polytopic membrane proteins. ${ }^{18}$ Recent studies demonstrated that Tom70 was essential for PTEN-induced kinase 1 (PINK1) import into mitochondria, ${ }^{19}$ which decreased the cardiac vulnerability to I/R injury. ${ }^{19,20}$ However, how MICU1 is translocated from cytosol to the inner mitochondrial membrane of cardiomyocyte and whether Tom70 is involved in this process remain unknown. Moreover, whether the expression/function of Tom70 is inhibited in ischemic/reperfused cardiomyocytes, thus impairing MICU1 localization, has not been previously investigated.

Therefore, the aims of present study are (1) to determine whether MI/R may alter mitochondrial localization of MICU1; if so, (2) whether it may contribute to mitochondrial dysfunction and MI/R injury; and (3) to identify the molecular mechanisms, particularly Tom70, controlling subcellular localization of MICU1.

\section{Results}

Mitochondrial MICU1 expression is downregulated following MI/R, and MICU1 deficiency aggravated MI/R injury. To determine the role of MICU1, a newly identified regulator of mitochondrial $\mathrm{Ca}^{2+}$ homeostasis, in $\mathrm{MI} / \mathrm{R}$, we first determined the total and mitochondrial MICU1 protein expression level following MI/R. Interestingly, I/R induced significant reduction in mitochondrial MICU1 expression, but not in total MICU1 expression, compared with sham-MI/R counterpart (Figure 1). To determine the functional significance of MICU1 inhibition following MI/R, mice models with cardiac MICU1 knockdown were successfully established by intramyocardial injection of MICU1-targeted small interfering RNA (siRNA; Supplementary Figures $1 \mathrm{~A}$ and B). Although MICU1 downregulation slightly increased mitochondrial $\mathrm{Ca}^{2+}$ content in cardiomyocytes, however it did not significantly disturb mitochondrial respiration, nor did it induce obvious myocardial injury (Supplementary Figures 1C-F). Moreover, we did not find any changes in basal physiological parameters in MICU1 knockdown mice (Table 1). Using ELISA kit, 2, 3, 5-triphenyltetrazolium chloride (TTC) staining and ultrasonography, we found that MICU1 deficiency markedly enhanced MI/R-induced cardiac troponin-I (cTnl) leak, MI and contractile dysfunction (Figures 2a and b and Supplementary Figure 2). The evaluation of myocardial apoptosis by terminal deoxynucleotidyltransferase-mediated dUTP nick end labeling (TUNEL) staining and caspace-3 activation revealed that downregulation of MICU1 aggregated MI/R-induced cardiomyocytes loss (Figure 2c). These results indicate that MICU1 is a cardioprotective molecule against $\mathrm{MI} / \mathrm{R}$ injury, whose mitochondrial expression is inhibited following MI/R. 
MICU1 inhibited mitochondrial $\mathrm{Ca}^{2+}$ overload and protected against mitochondrial morphological and functional impairment. The disruption of mitochondrial $\mathrm{Ca}^{2+}$ homeostasis is a key event in I/R-induced cellular damage. ${ }^{3,7}$ $\mathrm{Ca}^{2+}$ overload into the mitochondria results in depolarization of the inner mitochondrial membrane and suppression of mitochondrial energetics. ${ }^{21}$ We thus explored the effect of MICU1 on mitochondrial morphology and function. Consistent with previous studies, we found that mitochondrial $\mathrm{Ca}^{2+}$ content (assessed by atomic absorption flame spectroscopy) significantly increased in $\mathrm{Ml} / \mathrm{R}$. More importantly, MICU1 deficiency further aggravated mitochondrial $\mathrm{Ca}^{2+}$ overload,

Table 1 Physiological measurements

\begin{tabular}{lccccc}
\hline \multicolumn{5}{c}{ Basal physiological parameters in all kinds of mice } \\
\hline & Control & MICU1 KD & Tom70 KD & Tom70 sp & Tom70 sp+MICU1 KD \\
\hline HW (mg) & $121.0 \pm 5.4$ & $120.9 \pm 4.7$ & $119.5 \pm 5.9$ & $123.5 \pm 3.8$ & $126.4 \pm 6.7$ \\
BW (g) & $24.7 \pm 1.2$ & $23.7 \pm 2.1$ & $23.9 \pm 1.5$ & $24.7 \pm 2.3$ & $5.0 \pm 0.2$ \\
HW/BW (mg/g) & $4.9 \pm 0.2$ & $5.1 \pm 0.4$ & $5.0 \pm 0.3$ & $4.3 \pm 1.8$ & $4.2 \pm 0.5$ \\
HR (b.p.m.) & $410 \pm 36$ & $418 \pm 27$ & $421 \pm 39$ & $401 \pm 23$ & $68 \pm 45$ \\
LVEF (\%) & $71 \pm 3.8$ & $68 \pm 4.6$ & $40 \pm 3.5$ & $41 \pm 2.7$ & $39 \pm 3.5$ \\
LVFS (\%) & $43 \pm 2.3$ & $39 \pm 3.6$ & $40 \pm 4.2$ & & \\
\hline
\end{tabular}

Abbreviations: BW, body weight; $\mathrm{HR}$, heart rate; HW, heart weight; HW/BW, heart weight/body weight ratio; KD, knockdown; LVEF, left ventricle ejection fraction; LVFS, left ventricle fractional shortening; sp, supplementation

Cardiac weight index was defined as the ratio of heart weight/body weight (in $\mathrm{mg} / \mathrm{g}$ ). Cardiac function was assessed in anesthetized mice by transthoracic echocardiography

a

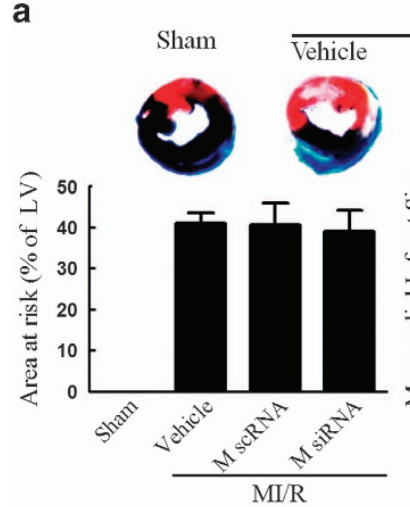

$\mathrm{MI} / \mathrm{R}$

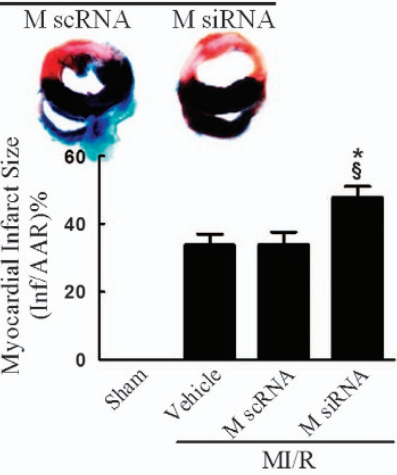

b
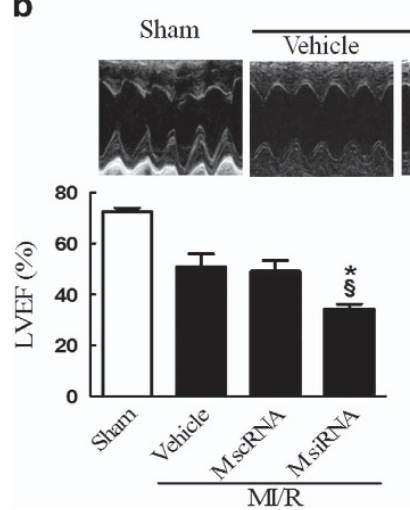

$\mathrm{MI} / \mathrm{R}$
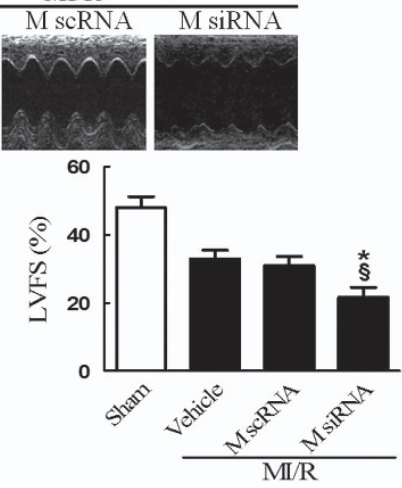

C
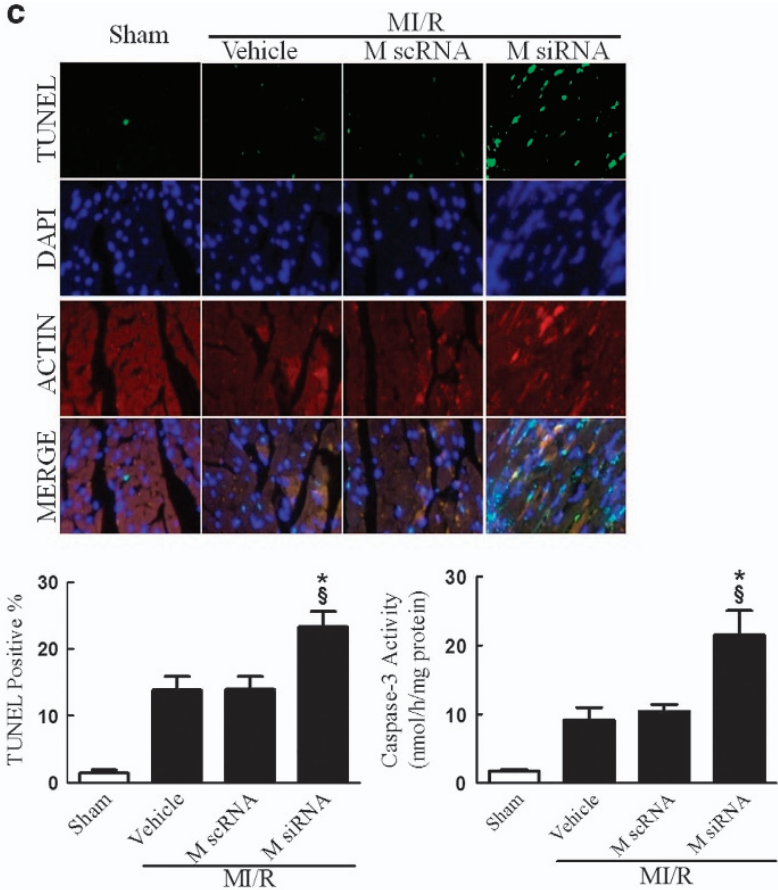

Figure 2 MICU1 deficiency worsened MI/R injury. (a) Myocardial infarct size was assessed by Evans blue/TTC double staining. The upper panel showed heart sections obtained from mice at $24 \mathrm{~h}$ after MI/R injury. Evans blue-stained areas (black) indicated non-ischemic/reperfused area; TTC-stained areas (red staining) indicated ischemic but viable tissue; Evans blue/TTC-staining-negative areas indicated infarct myocardium. The lower panels showed summary of area at risk (AAR) per left ventricle (LV) and infarct area (Inf) per AAR. (b) Cardiac function was assessed by echocardiography in mice $24 \mathrm{~h}$ after Ml/R injury. Representative M-mode images were shown in the upper panel. Left ventricle ejection fraction (LVEF) and fractional shortening (LVFS) were showed in the lower panels. (c) Myocardial apoptosis was determined by TUNEL staining in the upper panel. TUNEL staining (green) indicates apoptotic nuclei; DAPI counterstaining (blue) indicates total nuclei. TUNEL-positive nuclei were expressed as a percentage of the total number of nuclei, automatically counted and calculated by Image-Pro Plus software in the left of lower panel. Myocardial apoptosis was determined by caspase-3 activity assay in the right of lower panel. M scRNA, scrambled siRNA used as control; M siRNA, MICU1-specific siRNA. Presented values are means $\pm \mathrm{S} . \mathrm{E} . \mathrm{M}$. $N=6-8 /$ group. ${ }^{*} P<0.05$ versus vehicle of $M I / R$; ${ }^{\S} P<0.05$ versus $M$ scRNA of MI/R 
a
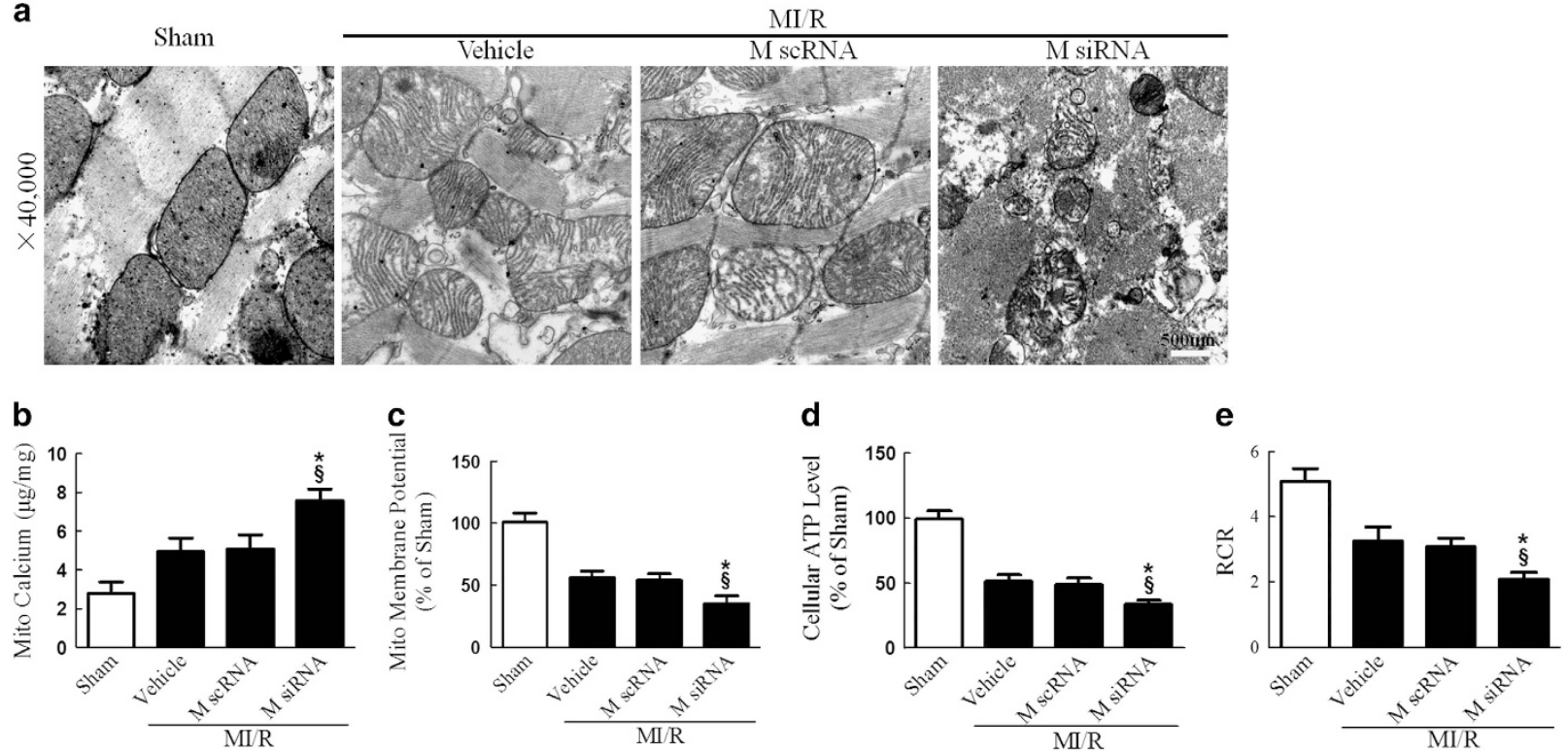

Figure 3 MICU1 deficiency aggravated MI/R-induced mitochondrial injury. (a) Representative mitochondrial morphologies detected by transmission electron microscopy. (b) Mitochondrial $\mathrm{Ca}^{2+}$ content was determined by atomic absorption flame spectroscopy $(\mu \mathrm{g} / \mathrm{mg})$. (c) Mitochondrial membrane potential was evaluated using JC-1 kit. (d) The ATP content of the myocardium was measured using a firefly luciferase-based ATP assay kit. (e) The respiratory control ratio (RCR) was assessed as the ratio of the state 3 to state 4 respiration. Mito, mitochondria; M scRNA, scrambled siRNA used as control; M siRNA, MICU1-specific siRNA. Presented values are means \pm S.E.M. $N=6-8 /$ group. ${ }^{*} P<0.05$ versus vehicle of $\mathrm{Ml} / \mathrm{R} ;{ }^{\S} P<0.05$ versus $\mathrm{M}$ scRNA of $\mathrm{Ml} / \mathrm{R}$

destructed mitochondrial morphology (evidenced by disappeared mitochondrial membranes integrity, unusual vesicle-like structures, completely unstructured cristae and ambiguous myofilaments), depressed ATP production and disturbed mitochondrial respiration (Figure 3 ). These results indicate that MICU1 inhibits post-MI mitochondrial $\mathrm{Ca}^{2+}$ overload, promoting structural integrity and subsequently metabolic function.

Mitochondrial MICU1 content is controlled by the importer receptor Tom70. Our results presented in Figure 1 demonstrated that mitochondrial, but not total, MICU1 is inhibited in cardiac tissue subjected to MI/R. To determine the mechanisms leading to selective inhibition of mitochondrial MICU1 level, the expression level of Tom70, a molecule responsible for initial recognition of mitochondrial pre-proteins from the cytosol, ${ }^{22}$ was determined in l/R heart. As illustrated in Figures $4 \mathrm{a} 1$ and b1, Tom70 expression, especially in cardiac mitochondria, was significantly inhibited following $30 \mathrm{~min}$ ischemia/3 $\mathrm{h}$ reperfusion. Notably, the reduction of mitochondrial Tom70 expression was found as early as 30 min reperfusion, while MICU1 was also proved to decrease as early as $1 \mathrm{~h}$ reperfusion (Supplementary Figure 3). To determine whether reduced Tom70 expression is responsible for decreased mitochondrial MICU1 expression, Tom70 expression was inhibited or elevated via intramyocardial injection of siRNA or Tom70-expressing lentivirus (Supplementary Figures 4 and 5). We did not find any changes in basal physiological parameters in both kinds of mice (Table 1). As illustrated in Figure 4b2, downregulation of Tom70 significantly reduced mitochondrial MICU1 content, evidenced by western blot. Moreover, during importing the inner membrane carrier proteins, it is an essential step that mitochondrial precursor proteins require initial recognition by Tom70 to promote the translocation. ${ }^{23}$ Thus, we investigated whether precursor MICU1 coordinated interaction with Tom70 by co-immunoprecipitation. As summarized in Figure 4a2, Tom70 successfully recognized precursor MICU1 in cardiac tissues. These results suggest that mitochondrial MICU1 localization is controlled by the importer receptor Tom70.

Tom70 deficiency accelerated MI/R injury. To further identify the role of Tom70 in MI/R injury, we investigated the effects of downregulation of Tom70 on cardiac injuries following I/R. We observed enhanced cTnl leak, enlarged MI size, suppressed cardiac function and increased myocardial apoptosis in Tom70-deficiency mice following MI/R (Figure 5 and Supplementary Figure 6). Moreover, we found that Tom70 deficiency deteriorated MI/R-induced destruction of mitochondrial morphology, depression of mitochondrial function and aggravation of mitochondrial $\mathrm{Ca}^{2+}$ overload (Figure 6). These results provide more evidences that Tom70 is a cardioprotective molecule against MI/R injury.

Tom70 overexpression markedly attenuated MI/R injury, which was partly abolished by MICU1 knockdown. To further determine the causal relationship between MICU1 and Tom70 in mitochondrial function and post-MI cardiac injury, we observed the functional consequence of Tom70 upregulation in $M I / R$ and the involvement of MICU1. As shown in Figures 7 and 8 and Supplementary Figure 7, upregulation of Tom70 significantly improved cardiac function, reduced $\mathrm{Ml}$ size, suppressed cTnl leak, decreased myocardial apoptosis, preserved mitochondrial morphology 
a Mouse cardiac tissues
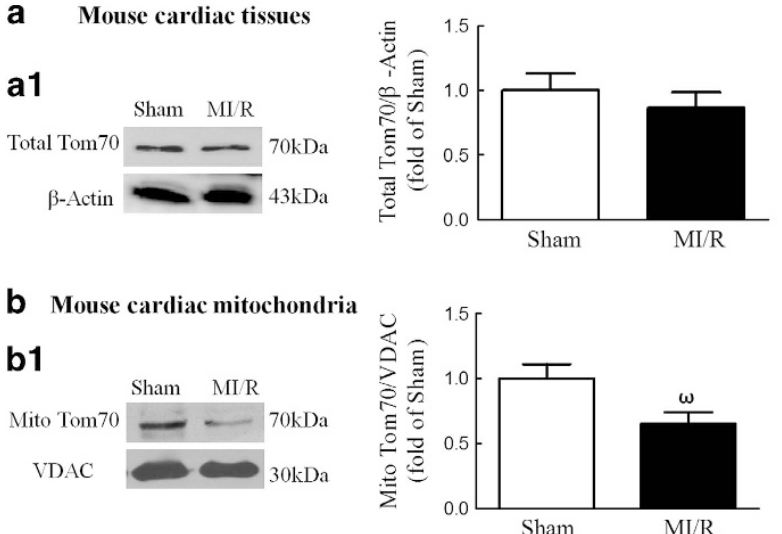

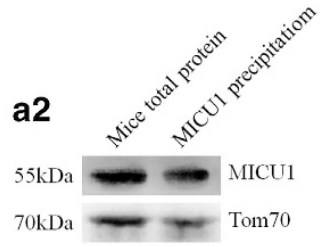

b2
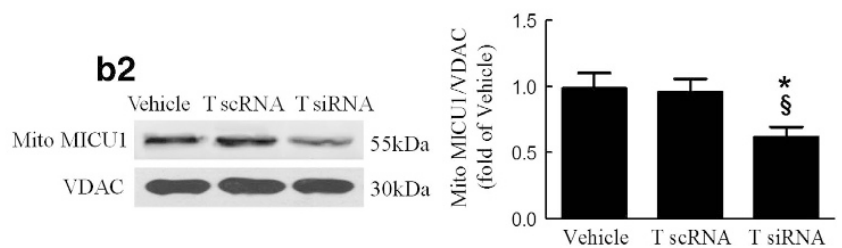

Figure 4 Mitochondrial MICU1 content was controlled by the importer receptor Tom70. (a1) Representative western blot images of total Tom70 and $\beta$-actin in the left panel; intensities of total Tom70 relative to $\beta$-actin in the right panel. (a2) Western blot analysis for MICU1 and Tom70 was performed on the precipitation of MICU1. (b1, b2) Representative western blot images showing mitochondrial Tom70 or MICU1 expression using VDAC as loading control in the left panel; intensities of mitochondrial Tom70 or MICU1 relative to VDAC in the right panel. Mito, mitochondria; T scRNA, scrambled siRNA used as control; T siRNA, Tom70-specific siRNA; VADC, voltage-dependent anion channel. Presented values are means \pm S.E.M. $N=6-8$ /group. ${ }^{\omega} P<0.05$ versus sham; ${ }^{*} P<0.05$ versus vehicle; ${ }^{\S} P<0.05$ versus T scRNA

a
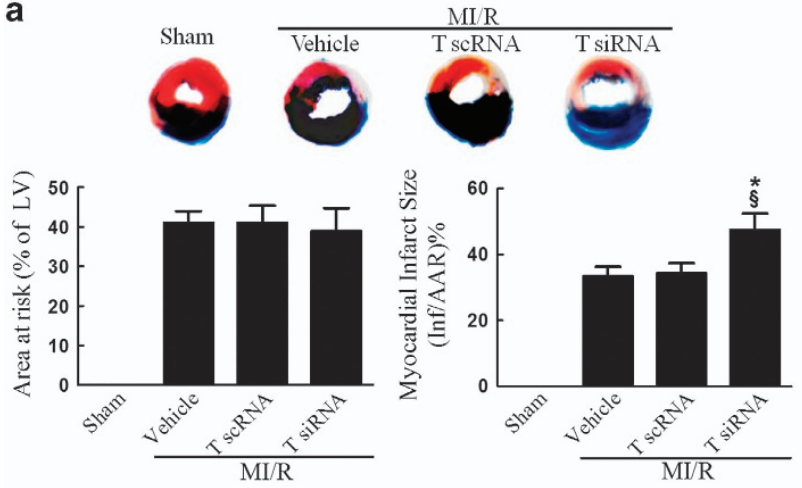

b

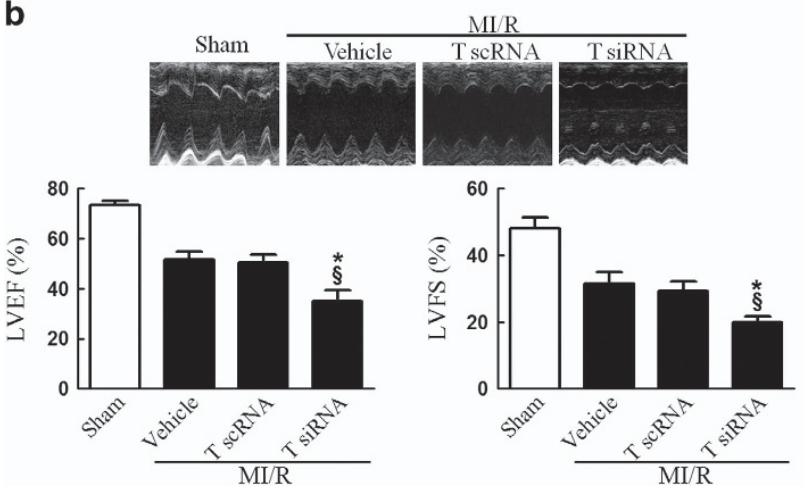

C
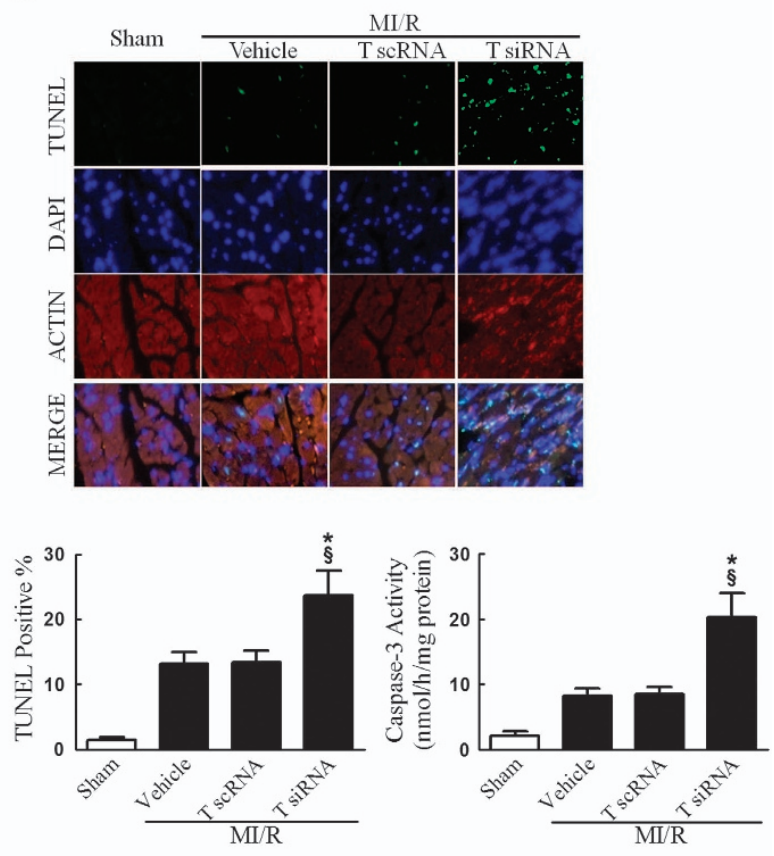

Figure 5 Tom70 deficiency worsened MI/R injury. (a) Myocardial infarct size assessed by Evans blue/TTC double staining. (b) Cardiac function was assessed by echocardiography. (c) Myocardial apoptosis was determined by TUNEL staining and caspase-3 activity assay. T scRNA, scrambled siRNA used as control; T siRNA, Tom70-specific siRNA. Presented values are means \pm S.E.M. $N=6-8 /$ group. ${ }^{*} P<0.05$ versus vehicle of $M l / R ;{ }^{~} P<0.05$ versus $\mathrm{T}$ scRNA of Ml/R

and improved mitochondrial function. Mechanistically, Tom70 supplementation successfully prevented mitochondrial $\mathrm{Ca}^{2+}$ overload (Figure 8b). However, the Tom70-mediated protection was largely abolished by MICU1 knockdown via intramyocardial injection of siRNA (Figures 7 and 8 and Supplementary Figure 7). These results reveal that MICU1 serves as an indispensable molecule in the protection of Tom70 against MI/R injury. Moreover, the protection provided by MICU1 overexpression was eliminated by Tom70 ablation (Supplementary Figure 8). These results indicate that MICU1 has protective function depending on Tom70 in MI/R. 
a

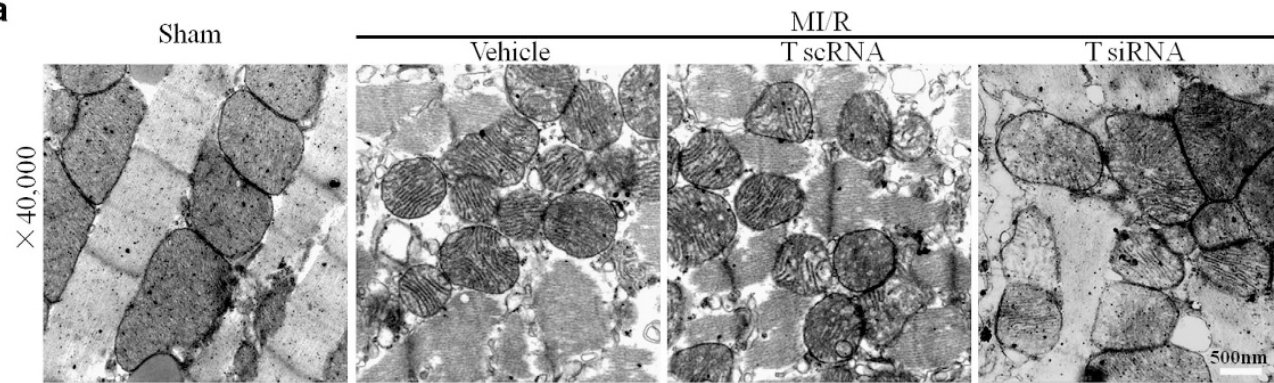

b

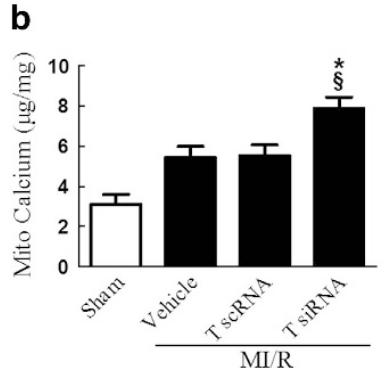

C

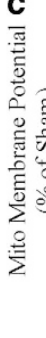

d

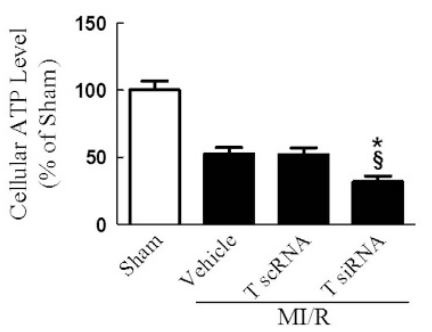

e

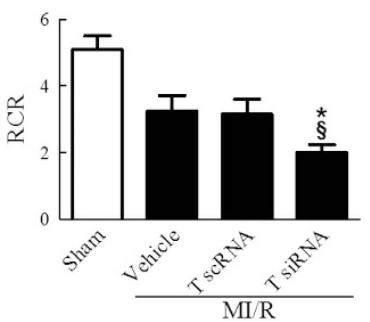

Figure 6 Tom70 deficiency aggravated MI/R-induced mitochondrial injury. (a) Representative mitochondrial morphologies detected by transmission electron microscopy. (b) Mitochondrial $\mathrm{Ca}^{2+}$ content was determined by atomic absorption flame spectroscopy $(\mu \mathrm{g} / \mathrm{mg})$. (c) Mitochondrial membrane potential evaluated by JC-1 kit. (d) The ATP content measured by ATP assay kit. (e) The respiratory control ratio (RCR) was determined as the ratio of the state 3 to state 4 respiration. T scRNA, scrambled siRNA used as control; T siRNA, Tom70-specific siRNA. Presented values are means \pm S.E.M. $N=6-8 /$ group. ${ }^{*} P<0.05$ versus vehicle of Ml/R; ${ }^{\S} P<0.05$ versus $\mathrm{T}$ scRNA of Ml/R

a

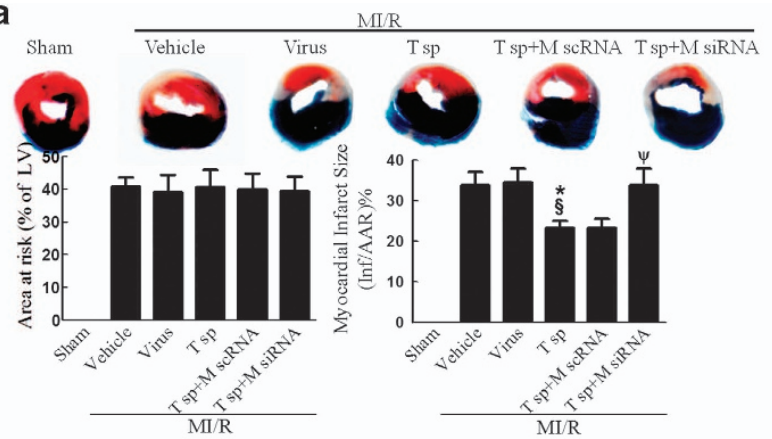

b

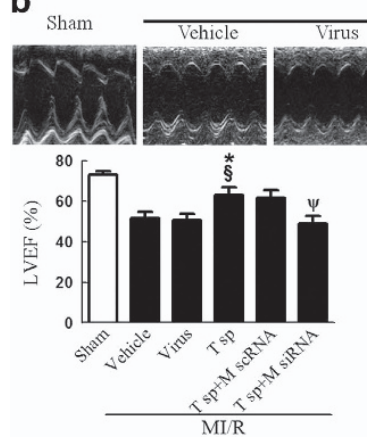

C
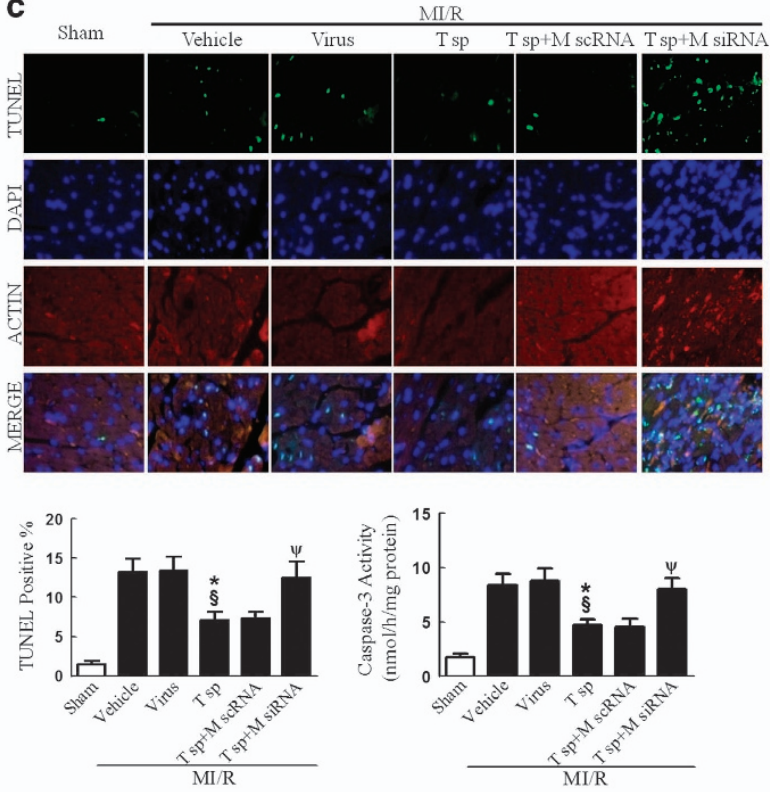

Figure 7 Tom70 supplementation attenuated MI/R injury, which was abolished by MICU1 ablation. (a) Myocardial infarct size assessed by Evans blue/TTC double staining. (b) Cardiac function was assessed by echocardiography. (c) Myocardial apoptosis was determined by TUNEL staining and caspase-3 activity assay. Mito, mitochondria; M scRNA, scrambled siRNA used as control; M siRNA, MICU1-specific siRNA; T sp, Tom70 supplementation; virus, lentivirus vector. Presented values are means \pm S.E.M. $N=6-8 /$ group. ${ }^{*} P<0.05$ versus vehicle of $\mathrm{Ml} / \mathrm{R} ;{ }^{\S} P<0.05$ versus virus of $\mathrm{Ml} / \mathrm{R} ;{ }^{\psi}{ }^{\psi} P<0.05$ versus (T sp+M scRNA) of Ml/R

\section{Discussion}

In the present study, we have made several novel observations. First, we provided the first evidence that although the total expression level of MICU1, a newly identified inhibitory regulator of $\mathrm{MCU}$, was unaltered; its mitochondrial localization was significantly disrupted in ischemic/reperfused heart. Second, we demonstrated that MICU1 exerted protection 

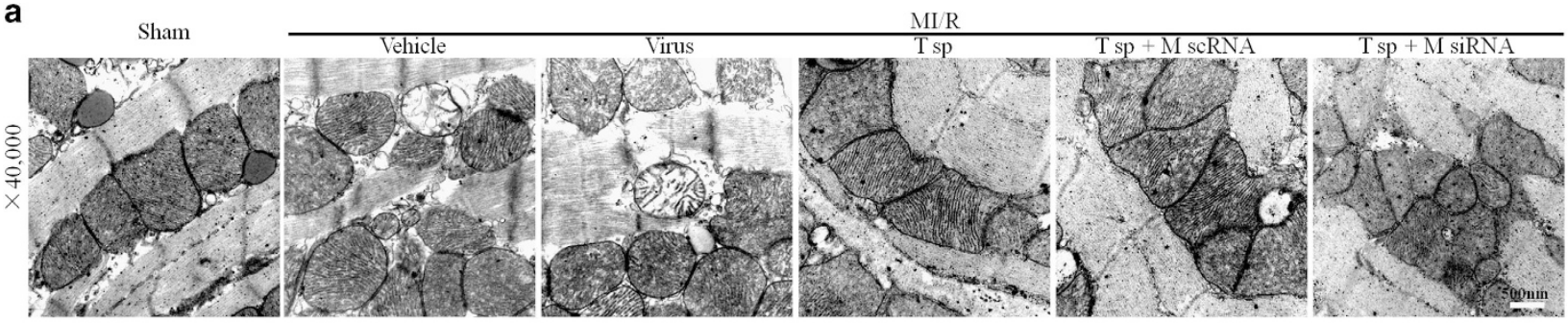

b

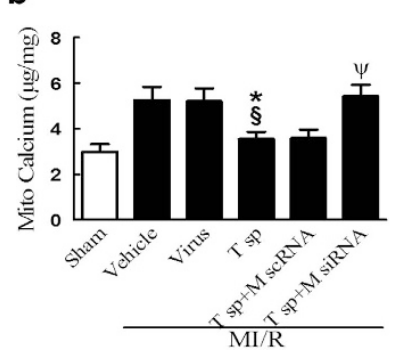

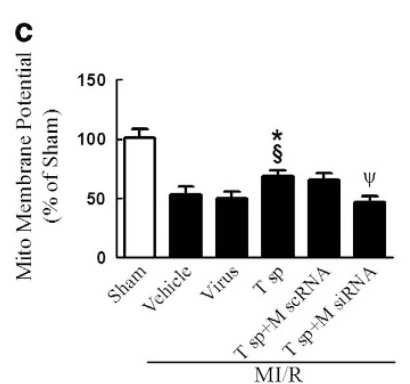

d

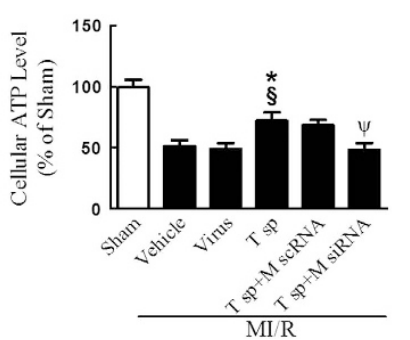

e

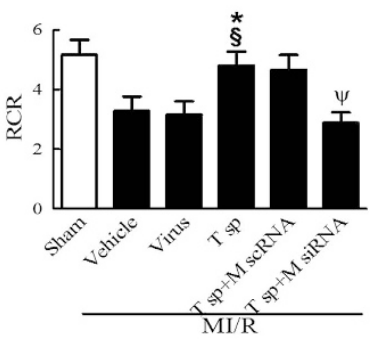

Figure 8 Tom70 supplementation attenuated MI/R-induced mitochondrial injury, which was reversed by MICU1 ablation. (a) Representative mitochondrial morphologies detected by transmission electron microscopy. (b) Mitochondrial $\mathrm{Ca}^{2+}$ content was determined by atomic absorption flame spectroscopy ( $\mu \mathrm{g} / \mathrm{mg}$ ). (c) Mitochondrial membrane potential evaluated by JC-1 kit. (d) The ATP content measured by ATP assay kit. (e) The respiratory control ratio (RCR) was evaluated as the ratio of the state 3 to state 4 respiration. Mito, mitochondria; M scRNA, scrambled siRNA used as control; M siRNA, MICU1-specific siRNA; T sp, Tom70 supplementation; virus, lentivirus vector. Presented values are means \pm S.E.M. $N=6-8 /$ group. ${ }^{*} P<0.05$ versus vehicle of Ml/R; ${ }^{\$} P<0.05$ versus virus of $M I / R ;{ }^{*} P<0.05$ versus (T sp+M scRNA) of MI/R

against $\mathrm{MI} / \mathrm{R}$ injury via prevention of mitochondrial $\mathrm{Ca}^{2+}$ overload, preservation of mitochondrial integrity and promotion of mitochondrial energy metabolism. Third, we found that the expression level of Tom70, the importer receptor having essential role in MICU1 mitochondrial localization, was downregulated after MI/R. Genetic manipulation revealed that loss of Tom70 decreased mitochondrial MICU1 localization and aggravated $\mathrm{MI} / \mathrm{R}$ injury; whereas, overexpression of Tom70 protected MI/R injury, which was eliminated by MICU1 ablation. Taken together, we have identified a novel cardioprotective pathway involving Tom70/MICU1 signaling, which represents a key regulatory machinery of mitochondrial $\mathrm{Ca}^{2+}$ homeostasis by the mitochondrial outer membrane protein transport system (Supplementary Figure 9).

As the cellular 'powerhouse' and 'apoptosis headquarter', mitochondria have critical roles in various physiological and pathological processes. ${ }^{24}$ As maintenance of $\mathrm{Ca}^{2+}$ gradients between cellular compartments depends on ATP-driven reactions, metabolic disruption by injurious stresses, such as $\mathrm{Ml} / \mathrm{R}$, quickly perturbs cellular $\mathrm{Ca}^{2+}$ homeostasis. In particular, release of $\mathrm{Ca}^{2+}$ from the endoplasmic reticulum may flood the cytosol with free $\mathrm{Ca}^{2+}$, leading to dysfunction of other organelles, particularly mitochondria. ${ }^{25}$ Dysregulation of $\mathrm{Ca}^{2+}$ homeostasis has long been implicated to predispose cell injury. Frey et al. ${ }^{26}$ revealed that $\mathrm{Ca}^{2+}$ overload led to cardiomyocyte death. In response to $\mathrm{Ca}^{2+}$ overload, both apoptosis and necrosis can contribute to cardiomyocyte loss by activating pro-death members of the Bcl2 family and opening the mPTP, respectively. ${ }^{27}$ An increase in mitochondrial $\mathrm{Ca}^{2+}$ content results in depolarization of the inner mitochondrial membrane, the production of reactive oxygen species and opening of the MPTP, a nonspecific pore in the inner mitochondrial membrane that is permeable to small molecules.

Mitochondrial $\mathrm{Ca}^{2+}$ uptake is mediated by the $\mathrm{Ca}^{2+}$ uniporter complex in the inner mitochondrial membrane termed MCU, a $\mathrm{Ca}^{2+}$-selective ion channel. ${ }^{14} \mathrm{MICU} 1$ was recently identified as a protein that localized to the inner mitochondrial membrane and suggested to be required for mitochondrial $\mathrm{Ca}^{2+}$ uptake regulation. ${ }^{13}$ MICU1 and MCU biochemically interact, being co-expressed across tissues and species. ${ }^{11,15}$ Mallilankaraman et al. ${ }^{16}$ found that MICU1 was required to preserve normal mitochondrial $\mathrm{Ca}^{2+}$ under basal conditions, and served as an essential inhibitory gatekeeper for MCU-mediated mitochondrial $\mathrm{Ca}^{2+}$ uptake that regulated cell survival. In our study we found that mitochondrial MICU1 expression was inhibited by $M I / R$, and MICU1 deficiency deteriorated mitochondrial $\mathrm{Ca}^{2+}$ overload and aggravated $\mathrm{MI} /$ $\mathrm{R}$ injury.

Strong evidences reveal that the Tom complex has critical role in the initial recognition of mitochondrial pre-proteins from the cytosol where the majority of mitochondrial proteins are synthesized and then transported into corresponding compartments in mitochondria. ${ }^{22}$ Many mitochondrial pre-proteins are targeted post-translationally, ${ }^{28}$ and requires initial recognition by import receptors. ${ }^{18,29}$ Tom70 contains a core region, ${ }^{28}$ within which four $\mathrm{C}$-terminal tetratricopeptide repeat motifs lie to recognize the internal targeting signals of polytopic membrane proteins. ${ }^{30}$ Moreover, Hsp70/Hsp90 chaperones have been reported to integrate the outer membrane-localized Tom70 receptor with mitochondrial preprotein targeting and translocation. ${ }^{28}$ Recently, Li et al..$^{31}$ revealed that Tom70 served as an importer receptor to govern the mitochondrial translocation of Opa1 and subsequently exhibited protective effects against pathological cardiac hypertrophy. What about 
the effect of Tom70 in the mitochondrial translocation of MICU1, particularly the change under MI/R stress? In our study, we found a significant reduction of mitochondrial Tom70 expression since $30 \mathrm{~min}$ reperfusion following $30 \mathrm{~min}$ ischemia, along with which mitochondrial MICU1 markedly decreased. Consistent with our results, Boengler et al. ${ }^{32}$ found that another import receptor of Tom complex Tom20 obviously decreased even after $10 \mathrm{~min}$ ischemia/15 min reperfusion. And then, we established Tom70 deficiency and supplementation mice models. As a result, we revealed that MI/R decreased Tom70 expression. Following Tom70 knockdown and supplementation, MI/R injury was aggravated or attenuated, respectively. These results indicate that altered Tom70 expression contributes to MI/R injury. Mechanistically, we demonstrated Tom70 deficiency decreased mitochondrial MICU1 localization and increased $\mathrm{Ca}^{2+}$ content. The protection provided by MICU1 overexpression was also eliminated by Tom70 ablation. These results indicate that mitochondrial MICU1 localization and function is restricted by Tom70. Moreover, Tom70 supplementation actually prevented mitochondrial $\mathrm{Ca}^{2+}$ overload, in which many factors regulated by Tom70 might have a role. The limitation of our study was hard to determine a specific effector for Tom70 supplementation, we guessed that MICU1 might participate in it. Utilizing cardiac tissues and co-immunoprecipitation, we found that Tom70 interacted with MICU1 in hearts. Thus, the mitochondrial translocation of MICU1 may be governed or facilitated by Tom70 in myocardium. More importantly, MICU1 ablation obviously eliminated the cardioprotection induced by Tom70 overexpression, revealing that MICU1 served as an essential factor to preserve Tom70's normal function in heart. Consistent with our results, Kato et al. ${ }^{19}$ found that Tom70 was essential for PINK1 import into mitochondria, which was reported to decrease the heart's vulnerability to $I / R$ injury. ${ }^{20}$ Here we revealed MICU1 as a new mitochondrial molecule participating in MI/R. Further studies elucidating the detailed mechanism of mitochondrial translocation of MICU1 by Tom70 receptor is currently undertaking.

\begin{abstract}
Materials and Methods Experimental protocols

Animals: C57BL6/J mice at the age of 10-12 weeks were used for the present study. All experiments were performed in adherence with the National institutes of Health Guidelines on the Use of Laboratory Animals, and were approved by the Fourth Military Medical University Committee on Animal Care. Mice were fed with standard laboratory animal chow with free access to tap water, and housed in a temperature- and humidity-controlled room with a $12 / 12 \mathrm{~h}$ light-dark cycle.
\end{abstract}

Reagent preparation: Lentivirus vectors were created as previously described. ${ }^{33}$ The Tom70a gene-coding sequence was amplified by PCR and subcloned into a lentivirus (LV4) expression plasmid V4886-2 vector to construct a lentivirus-based overexpression vector carrying the Tom70a sequence (V4886-2LV4-Tom70a), confirmed by PCR and DNA sequencing (GenePharma, Shanghai, China). Lentivirus expression plasmids were transfected into 293T cells to construct pLenti-GDI viral stock. The titers of the viral vectors used in this study were $1 \times 10^{9} \mathrm{TU} / \mathrm{ml}$. In additionally, the siRNAs against MICU1 and Tom70a were designed based on GenBank and purchased from GenePharma. MICU1-specific siRNA (against murine) contains four independent sequences: (1) sense $5^{\prime}$ GCUGGAGCAUCUCUUGAUATT-3' and antisense $5^{\prime}$-UAUCAAGAGAUGCUCC AGCTT-3'; (2) sense 5'-CUCCACUCCUCAGAGAAAUTT-3' and antisense $5^{\prime}$ AUUUCUCUGAGGAGUGGAGTT-3'; (3) sense 5'-UUGCCACCUUGAAAGUAA UTT-3' and antisense 5'-AUUACUUUCAAGGUGGCAATT-3'; and (4) sense 5'-
AGCCUUAUCCUGAGGACAATT-3' and antisense 5'-UUGUCCUCAGGAUAAG GCUTT-3'. Tom70a-specific siRNA (against murine) contains four independent sequences: (1) sense $5^{\prime}$-CAGGCAUAUACAGCAAACATT- 3 ' and antisense $5^{\prime}$-UGU UUGCUGUAUAUGCCUGTT-3'; (2) sense 5'-CCAGGCAUUAACAGAUCAATT-3' and antisense 5'-UUGAUCUGUUAAUGCCUGGTT-3'; (3) sense 5'-UGCUGU GUGUAUAUUAGAATT-3' and antisense 5'-UUCUAAUAUACACACAGCATT-3'; and (4) sense 5'-UGAGAAGAAUGUAGACCUUTT-3' and antisense 5'-AAGGUCUAC AUUCUUCUCATT-3'. Both siRNAs against MICU1 and Tom70a were cotransfected into $293 \mathrm{~T}$ cells to confirm that those designed sequences possessing knockdown efficiency, respectively.

In vivo delivery of siRNA and lentivirus of MICU1 and Tom70. A unit of $20 \mu \mathrm{g}$ of MICU1 siRNA, Tom70 siRNA or scrambled siRNA was diluted in $30 \mu$ l of vivo-jetPEI ${ }^{\mathrm{TM}}$ (Invitrogen, Carlsbad, CA, USA) and 10\% glucose mixture for preparation. Animals were first anesthetized with $2 \%$ isoflurane, and an incision was made between the fourth and fifth left ribs to expose the heart. A volume of $30 \mu \mathrm{l}$ of vehicle, scrambled siRNA, MICU1 siRNA or Tom70 siRNA solution were delivered via intramyocardial injection into the apex and anterolateral wall using a 30-gauge needle. Another group of mice were injected with $30 \mu \mathrm{l}$ of vehicle, $3.3 \times 10^{7} \mathrm{TU}$ V4886-2-LV4, $3.3 \times 10^{7}$ TU V4886-2-LV4-Tom70a, $\left(3.3 \times 10^{7}\right.$ TU V4886-2-LV4Tom70a+20 $\mu \mathrm{g}$ MICU1 scRNA) or $\left(3.3 \times 10^{7} \mathrm{TU}\right.$ V4886-2-LV4-Tom70a+20 $\mu \mathrm{g}$ MICU1 siRNA).

MI/R model. After $72 \mathrm{~h}$ of siRNA or lentivirus injection in hearts, the incision was opened under anesthetic condition once again. $\mathrm{Ml}$ was introduced by temporarily exteriorizing the heart via a left thoracic incision and placing a 6-0 silk suture slipknot around the left anterior descending coronary artery. After $30 \mathrm{~min}$ of $\mathrm{Ml}$, the slipknot was released, and the myocardium was reperfused for $3 \mathrm{~h}$ (for cell apoptosis, biochemical assays and mitochondrial morphology/function assessment) or $24 \mathrm{~h}$ (for cardiac function and infarct size determination). Sham-operated control mice (sham MI/R) underwent the same surgical procedures except that the suture placed under the left coronary artery was not tied. ${ }^{34}$

Measurement of cardiac function and myocardial infarct size. At the end of $24 \mathrm{~h}$ reperfusion, mice were re-anesthetized with isoflurane. Left ventricular ejection fraction and fractioning shortening were obtained by transthoracic echocardiography. After functional determination, the ligature around the coronary artery was retied, and myocardial infarct size was determined by the Evans blue/TTC (Sigma, St Louis, MO, USA) double-staining method, as described previously. ${ }^{35}$

Determination of cTnl. After $30 \mathrm{~min}$ of ischemia and $3 \mathrm{~h}$ of reperfusion, mice were completely exsanguinated. The circulating levels of specific isoform of cTnl were tested with an ELISA kit following the manufacturer's instructions (Life Diagnostics, West Chester, PA, USA). ${ }^{36}$

Determination of myocardial apoptosis. Myocardial apoptosis was determined by TUNEL staining (Roche, Nutley, NJ, USA). The paraffin-embedded tissue was cut into 4-5 $\mu$ m-thick sections. The sections were then incubated in $50 \mu \mathrm{l}$ of the TUNEL mixture $(47.5 \mu \mathrm{l}$ of TUNEL label containing fluorescein isothiocyanate-conjugated dUTP and $2.5 \mu \mathrm{l}$ of TUNEL enzyme) in a humidified chamber $\left(60 \mathrm{~min}, 37^{\circ} \mathrm{C}\right)$. Control sections were incubated with $50 \mu$ l of TUNEL label solution containing no TUNEL enzyme. An additional staining was performed with monoclonal anti- $\alpha$-sarcomeric actin (Sigma). All sections were photographed with a QICAM-Fast Digital Camera mounted atop an Olympus BX51 Fluorescence Microscope (Olympus America Inc, Center Valley, PA, USA). Total nuclei and the TUNEL-positive nuclei were counted by IP Lab Imaging Analysis Software (Version 3.5; Scanalytics, Fairfax, VA, USA). Apoptotic index (number of TUNEL-positive nuclei/total number of nuclei $\times 100$ ) was automatically calculated and exported to Microsoft Excel for further analysis. Cardiac caspase-3 activity was performed by using a caspase- 3 colorimetric assay kit (Keygen, Nanjing, China), following the manufacturer's instructions. Myocardial tissues were homogenized in ice-cold lysis buffer for $30 \mathrm{~s}$. The homogenates were centrifuged $\left(10000 \times g \times 5 \mathrm{~min}, 4^{\circ} \mathrm{C}\right)$ and the supernatants were collected. Supernatant containing $200 \mu \mathrm{g}$ of protein was loaded to each well of the 96-well plate and incubated with $25 \mu \mathrm{g} \mathrm{Ac-DEVD-pNA} \mathrm{at}$ $37^{\circ} \mathrm{C}$ for $1.5 \mathrm{~h}$. The pNA absorbance was quantified using a SpectraMax plate reader (SpectraMax, Atlanta, GA, USA) at $405 \mathrm{~nm}^{37}$ 
Isolation of fresh cardiac mitochondria. Cardiac tissues of IR region (area at risk, which was controlled by left anterior descending coronary artery, that is, the apex and anterolateral wall of the heart) were minced in mitochondria isolation buffer (250 mM sucrose, $10 \mathrm{mM}$ HEPES, $1 \mathrm{mM}$ EGTA and 0.5\% BSA, pH 7.4). The tissue was buffer-washed several times to remove blood and homogenized (Potter-Elvehjem). Subsequently, the lysates were centrifuged at $700 \times \mathrm{g}$ for $10 \mathrm{~min}$. The supernatant was filtered through a nylon filter of $250 \mathrm{~nm}$ and then centrifuged at $10780 \times g$ for $10 \mathrm{~min}$. The pellet was re-suspended in mitochondria isolation buffer and centrifuged at $7650 \times g$ for $10 \mathrm{~min}$. Again, the pellet was re-suspended in mitochondria isolation buffer, then layered on top of a $30 \%$ percoll solution in isolation buffer and centrifuged at $35000 \times g$ for $30 \mathrm{~min}$. The mitochondria was collected and washed twice in mitochondria isolation buffer by centrifugation at $7650 \times g$ for 5 min. $^{32}$

Immunoblotting. Cardiac tissues of IR region were lysed, sonicated and centrifuged. Mitochondria isolated from fresh cardiac tissues were lysed with lysis buffer $(25 \mathrm{mM}$ Tris- $\mathrm{HCl}, \mathrm{pH} 7.6,150 \mathrm{mM} \mathrm{NaCl}, 2 \mathrm{mM}$ EDTA, $1 \%$ sodium deoxycholate, $0.5 \%$ SDS, $1 \%$ Triton X-100 and protease inhibitor). After sonication, the lysates were centrifuged at $12000 \times g$ for $30 \mathrm{~min}$ to remove residue. Protein concentrations were measured using Bio-Rad kit (Bio-Rad Laboratories, Hercules, CA, USA). Proteins were separated by SDS-PAGE and then transferred to a polyvinylidenedifluoride membrane (Millipore, Bedford, MA, USA). After blocking with $5 \%$ skim milk in $1 \times$ PBST at room temperature for $1 \mathrm{~h}$, the membrane were incubated with primary antibody against MICU1 (1:500; Immunoway, CA, USA), Tom70 (1:500; Santa Cruz Biotechnology, Santa Cruz, CA, USA), $\beta$-actin (1:3000; Sigma) or VDAC (1:3000; Sigma) overnight at $4^{\circ} \mathrm{C}$. The membrane was then washed with $1 \times$ PBST and incubated with horseradish peroxidase-conjugated $\lg G$ antibody (Cell Signaling, Beverly, MA, USA) for $1 \mathrm{~h}$ at room temperature. The blots were visualized using a super signal chemiluminescence detection kit (Thermo Scientific, Waltham, MA, USA) and exposure to X-ray film. ${ }^{38}$

Immunohistochemistry and immunofluorescence staining. Myocardial tissues were fixed with $4 \%$ paraformaldehyde, and paraffin sections (3-5 $\mu \mathrm{m})$ were prepared for immunohistochemistry and immunofluorescence staining. For immunohistochemistry, the sections were blocked with $10 \%$ normal goat serum for $1 \mathrm{~h}$ and probed with anti-Tom70 antibody $\left(1: 20\right.$, Santa Cruz) at $4{ }^{\circ} \mathrm{C}$ overnight. Then they were incubated with biotinylated goat anti-mouse $\mathrm{lgG}$ at room temperature for $2 \mathrm{~h}$ and incubated with ExtrAvidin Peroxidase (Sigma/Aldrich Quimica, Madrid, Spain) for $1 \mathrm{~h}$. Positive staining was detected with $0.05 \% \mathrm{DAB} /$ $0.01 \% \mathrm{H}_{2} \mathrm{O}_{2}$ in $0.05 \mathrm{~mol} / \mathrm{l}$ Tris- $\mathrm{HCl}$ buffer. The negative control group, in which one of the primary antibodies was omitted and replaced with normal $\operatorname{lgG}$, showed no immunoreactivity. Nuclei were counterstained with 2-(4-amidino-phenyl)-6-indolecarba-midine dihydrochloride (DAPI). All photographs were taken under an Olympus BX51 Fluorescence Microscope (Olympus America Inc).

The spatial expression of MICU1 was evaluated with immunofluorescence using anti-MICU1 antibody at $4{ }^{\circ} \mathrm{C}$ overnight. All labeled sections were incubated with antibodies against MICU1 (1:50; Immunoway) in a humidified container at $4{ }^{\circ} \mathrm{C}$ overnight. After washing with PBS, the sections were incubated with tetramethylrhodamineisothiocyanate-conjugated second antibodies. After washing three times with PBS, DAPI (Beyotime, Shanghai, China) solution was added to stain the cell nucleus for 3 min. Sections were then washed in PBS and sealed with a coverslip. The slides were analyzed with laser confocal microscopy with excitation at $488 \mathrm{~nm}$ (Olympus, FV1000). Image J software (National Institutes of Health, Bethesda, MD, USA) was used for quantitative analysis, and total antibody staining was normalized to DAPI. ${ }^{39}$

Transmission electron microscopy. Hearts were removed and flushed in ice-cold PBS. Left ventricular walls were cut perpendicular to the long axis and trimmed into 1-2 mm-wide blocks. After fixing overnight in $4 \%$ glutaraldehyde, the sections were postixed in $1 \%$ osmium tetroxide for $1 \mathrm{~h}$, dehydrated using a graded ethanol immersion series and embedded in resin. Tissue pieces were cut into $80 \mathrm{~nm}$-thick sections by ultramicrotome (Leica, Vienna, Austria). The ultrathin sections were fixed on 200-slot grids coated with piloform membrane and observed with a JEM-1400 electron microscope (JEOL, Tokyo, Japan) and the micrographs were captured with CCD camera (OLYMPUE, Tokyo, Japan). Mitochondria were imaged at 40000 (40K) magnifications, and representative images were acquired as described previously. ${ }^{40}$
Evaluation of mitochondrial respiration. Mitochondrial respiration was tested at $25^{\circ} \mathrm{C}$ by using a Clark-type electrode connected to a respiration chamber (YSI Incorporated, Yellow Springs, $\mathrm{OH}, \mathrm{USA}$ ) and a linear chart recorder, in buffer (pH 7.4) containing $20 \mathrm{mM}$ HEPES, $10 \mathrm{mM} \mathrm{KCl}, 5 \mathrm{mM} \mathrm{KH} 2 \mathrm{PO} 4,2.5 \mathrm{mM} \mathrm{MgCl} 2$, $0.25 \mathrm{M}$ sucrose, $0.2 \mathrm{mM}$ EDTA and $1 \mathrm{mg} / \mathrm{ml}$ fatty acid-free BSA. Intact cardiac mitochondria were added to $0.5 \mathrm{mg}$ protein per $\mathrm{ml}$. For glutamate/malate respiration evaluation, substrates were added each at $5 \mathrm{mM}$. With the addition of $0.5 \mathrm{mM} \mathrm{ADP}$, state 3 respiration was tested. In the absence of ADP, state 4 respiration was determined. The respiratory control ratio was calculated as the ratio of the state 3 to state 4 respiration. ${ }^{41}$

Assessment of ATP content. The ATP content of the myocardium was measured using a firefly luciferase-based ATP assay kit (Beyotime) according to the manufacturer's instructions. Cardiac tissues were homogenized and centrifuged at $12000 \times g$ for $5 \mathrm{~min}$. Supernatants were mixed with ATP detection working dilution in a white 96-well plate. Luminance (RLU) was measured by using an Infinite M200 Microplate Reader (LabX, Midland, ON, Canada). Standard curves were also generated and the protein concentration of each treatment group was determined using the Bradford protein assay. Total ATP levels were expressed as nmol/mg protein. ${ }^{42}$

Measurement of mitochondrial membrane potential $(\Delta \Psi \mathrm{m})$. The quantification of mitochondrial potential variation was evaluated using JC-1 (Sigma, Taufkirchen, Germany). Cardiomyocytes ( $\approx 50$ cells from 8 mice per group) isolated from mice were seeded on gelatin-coated culture chamber slides and stained with JC-1 $(5 \mu \mathrm{mo} / / \mathrm{l})$ at $37^{\circ} \mathrm{C}$ for $10 \mathrm{~min}$. Cells were rinsed with the HEPES-saline buffer. Fluorescence of each sample was read at excitation wavelength of $490 \mathrm{~nm}$ and emission wavelength of 530 and $590 \mathrm{~nm}$ using a spectrofluorimeter (SpectraMax Gemini XS, SpectraMax). In healthy cells, a high concentration of JC-1 forms aggregates that yield red fluorescence at $\approx 590 \mathrm{~nm}$. In unhealthy cells, JC-1 exists as a monomer at low concentration emitting red fluorescence at $\approx 530 \mathrm{~nm}$. Results in fluorescence intensity were expressed as the ratio of 590 to $530 \mathrm{~nm}$ emission. ${ }^{43}$

Determination of mitochondrial $\mathrm{Ca}^{2+}$ content by atomic absorption flame spectroscopy. Mitochondrial samples for atomic absorption for quantification of calcium were dried at $200^{\circ} \mathrm{C}$ in an oven for $2 \mathrm{~h}$, weighed in its entirety and hydrolyzed in glass tubes containing $1 \mathrm{ml}$ of $6 \mathrm{M}$ hydrochloric acid. The tubes were heated to $92^{\circ} \mathrm{C}$ in a water bath. The hydrolyzed samples were then sent for spectrometry analysis (PERKIN ELMER 4100, LACTEC-PR, Brazil), and the results were expressed in microgram of calcium per microgram of tissue mitochondrial proteins. ${ }^{44}$

Immunoprecipitation of MICU1 with Tom70. MICU1 antibody was bound to pre-washed protein A/G Plus Agarose (Santa Cruz Biotechnology, Santa Cruz, CA, USA). The beads were then blocked with $1 \mathrm{mg} \mathrm{BSA}$ and washed once with IP buffer ( $150 \mathrm{mM} \mathrm{NaCl}, 1.5 \mathrm{mM} \mathrm{MgCl} 2,1 \mathrm{mM}$ EGTA and $20 \mathrm{mM}$ HEPES ( $\mathrm{pH}$ 7.4) buffer containing $1 \%$ NP40). The diluted myocardial extracts were added to the beads and rotated at $4{ }^{\circ} \mathrm{C}$ for $2 \mathrm{~h}$. The beads were washed three times with IP buffer and extracted with $20 \mu \mathrm{l}$ of $2 \times$ sample buffer containing $20 \mathrm{mM}$ DTT. The eluates were then subjected to western blot analysis.

Statistical analysis. All values are expressed as mean \pm S.E.M. and obtained from six to eight separate experimental preparations. Data (except western blot density) were subjected to ANOVA followed by Bonferroni correction for post hoc $t$-test. Western blot densities were analyzed with the Kruskal-Wallis test followed by Dunn post hoc test. $P<0.05$ was considered statistically significant.

\section{Conflict of Interest}

The authors declare no conflict of interest.

Acknowledgements. This work was supported by Program for National Science Fund for Distinguished Young Scholars of China (Grant No. 81225001), National Key Basic Research Program of China (973 Program, Grant No. 2013CB531204), New Century Excellent Talents in University (Grant No. NCET-11. 0870), Program for Changjiang Scholars and Innovative Research Team in University (Grant No. PCSIRT1053), Key Science and Technology Innovation Team in Shaanxi Province (Grant No. 2014KCT-19), National Science Funds of China (Grants No. 81500208, 81170186 and 81470478), China Postdoctoral Science Foundation (Grant 
No. 2017M613429), Science Fund for Distinguished Young Scholars of Sichuan Province (Grant No. 2017JQ0012), Science \& Technology Project of Sichuan Province (Grant No. 2015JY0277), Major Science and Technology Project of China 'Significant New Drug Development' (Grant No. 2012ZX09J12108-06B) and The Key Technologies R \& D Program of Shannxi Province (Grant No. 2015SF096).

1. Zhang $Y$, Ren J. Targeting autophagy for the therapeutic application of histone deacetylase inhibitors in ischemia/reperfusion heart injury. Circulation 2014; 129: 1088-1091.

2. Xie M, Kong Y, Tan W, May H, Battiprolu PK, Pedrozo Z et al. Histone deacetylase inhibition blunts ischemia/reperfusion injury by inducing cardiomyocyte autophagy. Circulation 2014: 129: $1139-1151$

3. Murphy E, Steenbergen C. Mechanisms underlying acute protection from cardiac ischemiareperfusion injury. Physiol Rev 2008; 88: 581-609.

4. Solaini G, Harris DA. Biochemical dysfunction in heart mitochondria exposed to ischaemia and reperfusion. Biochem J 2005; 390: 377-394.

5. Kubli DA, Gustafsson AB. Mitochondria and mitophagy: the yin and yang of cell death control. Circ Res 2012; 111: 1208-1221.

6. Balaban $\mathrm{RS}$. The role of $\mathrm{Ca}(2+)$ signaling in the coordination of mitochondrial ATP production with cardiac work. Biochim Biophys Acta 2009; 1787: 1334-1341.

7. Aurora AB, Mahmoud Al, Luo X, Johnson BA, van Rooij E, Matsuzaki S et al. MicroRNA-214 protects the mouse heart from ischemic injury by controlling $\mathrm{Ca}(2)(+)$ overload and cell death. J Clin Invest 2012; 122: 1222-1232.

8. Liu T, Takimoto E, Dimaano VL, DeMazumder D, Kettlewell S, Smith G et al. Inhibiting mitochondrial $\mathrm{Na}+/ \mathrm{Ca} 2+$ exchange prevents sudden death in a Guinea pig model of heart failure. Circ Res 2014; 115: 44-54.

9. Brookes PS, Yoon Y, Robotham JL, Anders MW, Sheu SS. Calcium, ATP, and ROS: a mitochondrial love-hate triangle. Am J Physiol Cell Physiol 2004; 287: C817-C833.

10. Marchi S, Pinton P. The mitochondrial calcium uniporter complex: molecular components, structure and physiopathological implications. J Physiol 2014; 592: 829-839.

11. De Stefani $D$, Raffaello A, Teardo E, Szabo I, Rizzuto R. A forty-kilodalton protein of the inner membrane is the mitochondrial calcium uniporter. Nature 2011; 476: 336-340.

12. Luongo TS, Lambert JP, Yuan A, Zhang X, Gross $P$, Song J et al. The mitochondrial calcium uniporter matches energetic supply with cardiac workload during stress and modulates permeability transition. Cell Rep 2015; 12: 23-34.

13. Perocchi F, Gohil VM, Girgis HS, Bao XR, McCombs JE, Palmer AE et al. MICU1 encodes a mitochondrial EF hand protein required for $\mathrm{Ca}(2+)$ uptake. Nature 2010; 467: 291-296.

14. Logan CV, Szabadkai G, Sharpe JA, Parry DA, Torelli S, Childs AM et al. Loss-of-function mutations in MICU1 cause a brain and muscle disorder linked to primary alterations in mitochondrial calcium signaling. Nat Genet 2014; 46: 188-193.

15. Baughman JM, Perocchi F, Girgis HS, Plovanich M, Belcher-Timme CA, Sancak $Y$ et al. Integrative genomics identifies MCU as an essential component of the mitochondrial calcium uniporter. Nature 2011: 476: 341-345.

16. Mallilankaraman K, Doonan P, Cardenas C, Chandramoorthy HC, Muller M, Miller R et al. MICU1 is an essential gatekeeper for MCU-mediated mitochondrial $\mathrm{Ca}(2+)$ uptake that regulates cell survival. Cell 2012; 151: 630-644.

17. Baker MJ, Frazier AE, Gulbis JM, Ryan MT. Mitochondrial protein-import machinery: correlating structure with function. Trends Cell Biol 2007; 17: 456-464.

18. Yamamoto H, Fukui K, Takahashi H, Kitamura S, Shiota T, Terao K et al. Roles of Tom70 in import of presequence-containing mitochondrial proteins. J Biol Chem 2009; 284: 31635-31646.

19. Kato H, Lu Q, Rapaport D, Kozjak-Pavlovic V. Tom70 is essential for PINK1 import into mitochondria. PLOS ONE 2013; 8: e58435.

20. Siddall HK, Yellon DM, Ong SB, Mukherjee UA, Burke N, Hall AR et al. Loss of PINK1 increases the heart's vulnerability to ischemia-reperfusion injury. PLOS ONE 2013; 8: e62400.

21. Weiss JN, Korge $P$, Honda HM, Ping P. Role of the mitochondrial permeability transition in myocardial disease. Circ Res 2003; 93: 292-301.

22. Neupert W, Herrmann JM. Translocation of proteins into mitochondria. Annu Rev Biochem 2007; 76: 723-749

23. Fan AC, Kozlov G, Hoegl A, Marcellus RC, Wong MJ, Gehring K et al. Interaction between the human mitochondrial import receptors Tom20 and Tom70 in vitro suggests a chaperone displacement mechanism. J Biol Chem 2011; 286: 32208-32219.

24. Hollander JM, Thapa D, Shepherd DL. Physiological and structural differences in spatially distinct subpopulations of cardiac mitochondria: influence of cardiac pathologies. Am J Physiol Heart Circ Physiol 2014; 307: H1-14.

25. Orrenius S, McConkey DJ, Bellomo G, Nicotera P. Role of Ca2+ in toxic cell killing. Trends Pharmacol Sci 1989; 10: 281-285.
26. Frey N, McKinsey TA, Olson EN. Decoding calcium signals involved in cardiac growth and function. Nat Med 2000; 6: 1221-1227.

27. Dorn GW 2nd. Apoptotic and non-apoptotic programmed cardiomyocyte death in ventricular remodelling. Cardiovasc Res 2009; 81: 465-473.

28. Young JC, Hoogenraad NJ, Hartl FU. Molecular chaperones Hsp90 and Hsp70 deliver preproteins to the mitochondrial import receptor Tom70. Cell 2003; 112: 41-50.

29. Yamano K, Yatsukawa Y, Esaki M, Hobbs AE, Jensen RE, Endo T. Tom20 and Tom22 share the common signal recognition pathway in mitochondrial protein import. J Biol Chem 2008; 283: 3799-3807.

30. Lithgow T, Glick BS, Schatz G. The protein import receptor of mitochondria. Trends Biochem Sci 1995; 20: 98-101.

31. Li J, Qi M, Li C, Shi D, Zhang D, Xie D et al. Tom70 serves as a molecular switch to determine pathological cardiac hypertrophy. Cell Res 2014; 24: 977-993.

32. Boengler K, Gres $P$, Cabestrero A, Ruiz-Meana M, Garcia-Dorado D, Heusch G et al. Prevention of the ischemia-induced decrease in mitochondrial Tom20 content by ischemic preconditioning. J Mol Cell Cardiol 2006; 41: 426-430.

33. Kobinger GP, Weiner DJ, Yu QC, Wilson JM. Filovirus-pseudotyped lentiviral vector can efficiently and stably transduce airway epithelia in vivo. Nat Biotechnol 2001; 19: 225-230.

34. Gao E, Lei YH, Shang X, Huang ZM, Zuo L, Boucher M et al. A novel and efficient model of coronary artery ligation and myocardial infarction in the mouse. Circ Res 2010; 107: $1445-1453$.

35. Pei H, Yu Q, Xue Q, Guo Y, Sun L, Hong Z et al. Notch1 cardioprotection in myocardial ischemia/reperfusion involves reduction of oxidative/nitrative stress. Basic Res Cardiol 2013; 108: 373.

36. Elrod JW, Calvert JW, Morrison J, Doeller JE, Kraus DW, Tao L et al. Hydrogen sulfide attenuates myocardial ischemia-reperfusion injury by preservation of mitochondrial function. Proc Natl Acad Sci USA 2007; 104: 15560-15565.

37. Pei H, Qu Y, Lu X, Yu Q, Lian K, Liu P et al. Cardiac-derived adiponectin induced by long-term insulin treatment ameliorates myocardial ischemia/reperfusion injury in type 1 diabetic mice via AMPK signaling. Basic Res Cardiol 2013; 108: 322

38. Liu S, Yin T, Wei X, Yi W, Qu Y, Liu Y et al. Downregulation of adiponectin induced by tumor necrosis factor alpha is involved in the aggravation of posttraumatic myocardial ischemia/ reperfusion injury. Crit Care Med 2011; 39: 1935-1943.

39. Li T, Hu J, He GH, Li Y, Zhu CC, Hou WG et al. Up-regulation of NDRG2 through nuclear factor-kappa $B$ is required for Leydig cell apoptosis in both human and murine infertile testes. Biochim Biophys Acta 2012; 1822: 301-313.

40. Liu L, Feng D, Chen G, Chen M, Zheng Q, Song P et al. Mitochondrial outer-membrane protein FUNDC1 mediates hypoxia-induced mitophagy in mammalian cells. Nat Cell Biol 2012; 14: 177-185.

41. Zhou HZ, Ma X, Gray MO, Zhu BQ, Nguyen AP, Baker AJ et al. Transgenic MMP-2 expression induces latent cardiac mitochondrial dysfunction. Biochem Biophys Res Commun 2007; 358: 189-195

42. Yue R, Hu H, Yiu KH, Luo T, Zhou Z, Xu L et al. Lycopene protects against hypoxia/ reoxygenation-induced apoptosis by preventing mitochondrial dysfunction in primary neonatal mouse cardiomyocytes. PLOS ONE 2012; 7: e50778.

43. Zhang S, Liu X, Bawa-Khalfe T, Lu LS, Lyu YL, Liu LF et al. Identification of the molecular basis of doxorubicin-induced cardiotoxicity. Nat Med 2012; 18: 1639-1642.

44. Collatusso C, Roderian JG, Vieira ED, Costa FD, Noronha L, Fornazari Dde F. Effect of SDS-based decelullarization in the prevention of calcification in glutaraldehyde-preserved bovine pericardium: study in rats. Rev Bras Cir Cardiovasc 2012; 27: 88-96.

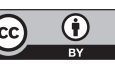

Cell Death and Disease is an open-access journal published by Nature Publishing Group. This work is licensed under a Creative Commons Attribution 4.0 International License. The images or other third party material in this article are included in the article's Creative Commons license, unless indicated otherwise in the credit line; if the material is not included under the Creative Commons license, users will need to obtain permission from the license holder to reproduce the material. To view a copy of this license, visit http://creativecommons.org/licenses/by/4.0/

(C) The Author(s) 2017 